\title{
DIGITAL TWIN: DECREASING THE COGNITIVE LOAD BY USING INDUSTRY 4.0 SIMULATION
}

\author{
Lukas Heschl, Thomas Dilger, Reinhard Bernsteiner and Christian Ploder \\ MCI - Management Center Innsbruck \\ Universitätsstrasse 15, 6020 Innsbruck, Austria
}

\begin{abstract}
As the integration of teaching theory and the application of Digital Twin (DT) technology is a challenge in our study programs, the authors put an effort on finding a supporting industry simulation model for in-class use. With the Fischertechnik Industry 4.0 Simulation Unit. This paper describes the objectives and the theoretical background of why this would be used in a case-based teaching setting. Further on, the authors are interested in the measurement of the cognitive load for the students and the influence of better design the lectures based on the simulation. The methodology is clearly stated and it is described how the first implementation of the given scenario in October 2020 will be done.
\end{abstract}

\section{KEYWORDS}

Digital Twin, Case-Based Teaching, Simulation, Augmented Reality, Cognitive Load Measurement

\section{INTRODUCTION}

Industry 4.0 is an umbrella term promoted by the German government in 2011 to increase the digital transformation of manufacturing (Fuchs, 2018). This strategy was put forward to act on the new generation of information technologies as well as its integration and extensive applications (such as the Internet of Things - IoT, Big Data, Social Media, Cloud Computing, Sensors, and Artificial Intelligence which are used for physical goods). Furthermore, the purpose of this work is to achieve interoperability between the virtual and physical world of manufacturing. The communication and interaction of both fields can be one of the bottlenecks to accomplish this purpose (Tao, Zhang, Cheng, \& Qi, 2017).

These virtual products are defined by Grieves (2015) as a depiction of products that are identical to their physical counterpart. Before a wide variety of sensing technologies digitalized data-collection, the rise of Manufacturing Execution Systems (MES) resulted in a richness of data collection (Grieves, 2015) which can also be seen in the current discussions about Low Code / No Code applications (Ploder et al., 2019). Through this technological progress, the concept of Digital Twin (DT) moved from an exciting approach to understanding the product with its environmental information to a demanding element of an enterprise-wide closed-loop product lifecycle. Fully implemented within a company, it can help to reduce costs and develop innovation for the quality of manufactured products (Grieves, 2015).

For students it can be hard to understand IT systems supporting an enterprise-wide product lifecycle. By creating an integrated learning scenario with the support of simulation, the hands-on experience will help to understand this big picture. With the Fischertechnik Industry 4.0, it is possible to simulate and present networked digital applications in a real production environment. This simulation enables us to digitalize the ordering, production, and delivery process. Through a network, the generated data of environmental sensors are synchronized to an IoT-Platform. With this data, a DT can be created and will help to decrease the cognitive load on students to understand this big picture of product lifecycles and Industry 4.0. 


\section{THEORETICAL BACKGROUND}

One of the key drivers in Industry 4.0 is the digital transformation of value chains, as well as the creation of digital products. As a result of this digitalization, new business models can be created. The usage of technologies in Industry 4.0 is meant to gain an advantage from the high amount of collected data. With analysis and simulations, the collected data can be used for a better understanding of the limitations and environmental aspects of a product or process.

The digital transformation in companies with cloud-based technologies, high-performance computing, or the IoT led to the possibility of imitating and modeling the physical world. The DT is a virtual replica that enables advanced and complex simulation, which is used to understand the performance of the product in the present and future. With data collected from connected sensors combined with related information, predictive analysis can be performed. The technology of the DT is a combination of artificial intelligence, data analytics, and IoT. As a result of the increasing networking and acquisition of data by sensors, more and more data are being collected. This data can also be used in new ways to execute the DT approaches, allow the development of new digital products and consequently affect other areas of technology, such as the collection of large amounts of data.

\subsection{Digital Twin}

The concept of the DT was introduced in 2003 at the University of Michigan in the course of product life cycle management (PLM) (Grieves, 2015). Its vision includes the full physical and functional description of components, products, or systems (Boschert \& Rosen, 2016). The approach of using twins is originated in the Apollo program of NASA. Two identical space vehicles were built to simulate conditions during the mission and mirror them in the twin vehicle that stayed on earth. In this understanding, a prototype to reflect the real operating terms for simulation can be defined as a twin (Rosen, von Wichert, Lo, \& Bettenhausen, 2015).

Following this idea to digitalize all life cycles of the physical model into a complete digital model is the vision of a DT. In the last decades, simulation developed from a restricted technology for experts to a standard tool for engineers in their daily tasks and as a core function of systems (Boschert \& Rosen, 2016). Prototyping and simulations to develop is nowadays a standard process to validate requirements and discover problems in advance and, hence, to realize failure prediction. Simulation is a conjunction of all life cycles in the physical and virtual world (Boschert \& Rosen, 2016).

The core functionality of a DT is provided through the collected data from the physical model. In the significant data-driven manufacturing era at any stage of the product lifecycle, data is processed, gathered, and leads to increased creation of digital artifacts (Tao et al., 2018; Rosen et al., 2015). The objects can be seen as stored data, containing all information that is needed by different stakeholders, but the DT is more than a data storage. It has a structure where all components are connected (Rosen et al., 2015). From a technical point of view, the data volume is too vast, diverse, and unstructured (Boschert \& Rosen, 2016). The product lifecycle is an iterative process, and as a consequence, lots of duplicate data in several phases can cause a waste of resources and lead to sharing problems (Tao et al., 2018). Grieves (2015) demonstrates three tools of human knowledge, which are supported by the capabilities of DT: (1) Conceptualization, (2) Comparison, and (3) Collaboration.

Conceptualization reflects that humans take in all data about a situation and then conceptualize it in their mind. During processing this visual information, it will be reduced to letters and symbols and then re-conceptualized visually. A loss of details and time is the result of this mechanism. The concept of DT eliminates this counterproductive mental step and builds a collective mindset of physical and virtual product information. As an alternative to looking at a bunch of numbers, the DT allows us to look at the progress of the physical product (Grieves, 2015).

Comparison is a natural habit of human beings, and it is done unconsciously and continuously with the desired and actual result to determine a difference. Working out the differences between physical and virtual product information is very inefficient. With a DT in place, the comparison of the ideal character with the actual measurement can be made quickly. Are the difference highlighted in colors, instantaneous decisions can be made (Grieves, 2015).

Collaboration brings more variability and perspectives on situations. Furthermore, the DT allows a shared model of conceptualization and solves the problem of individual concepts. To be at the same location is not 
needed anymore. The capabilities of DT additionally allow viewing physical products with overlaying their virtual counterpart at any stage in the factory, which could be extended across multiple factories across the globe (Grieves, 2015). The merge of information about how the product is manufactured and built can bring a contemporary perspective of how the process is meeting its specification goals. With this real-time information, digital factories can be created to predict how the product is to be manufactured. Emphasizing the connection between physical and virtual products will increase efficiency, the consistency and lead to higher quality of the product (Grieves, 2015).

\subsection{Industrie 4.0 Simulation Unit}

The Fischertechnik Simulation Units aim to create a factory simulation for a whole production chain with its modules and controller units that are connected and transfer their data to the cloud. Therefore, it allows us to develop a process that acts as a training model. It consists of an automated high-bay warehouse with a vacuum gripper robot, a multi-processing station with an oven, and a sorting line with detection. By connecting and controlling these stations, a complete production line can be demonstrated. The controllers are programmable with ROBO Pro, C-Compiler, PC-Library, and are preinstalled with similar programs.

In addition, through an environmental sensor and a pivoting camera, values of temperature, humidity, air pressure, and air quality are synchronized with the IoT-Platform via an MQTT broker. MQTT is based on industry standards, and all data can be immediately seen on the dashboard and used for further analysis. The traceability of the current status is enabled through the unique identification number of each workpiece.

The capabilities of smart and connected products and their resulting data are altering every activity in a manufacturing value chain. Traditional sources of data across the value chain are being supplemented by the product itself (Porter \& Heppelmann, 2015). Handling a lot of information brings up the need to develop Human-Computer Interfaces for the DT, to overcome the problem of showing the necessary information to the right user (Schroeder et al., 2016). Therefore, new digital user interfaces are enabled and eliminate the need of controlling the product itself (Porter \& Heppelmann, 2015). Augmented Reality as a critical enabling technology can display digital content onto the real world by using mobile devices that have proven to be accessible and highly versatile (Schroeder et al., 2016). Furthermore, Augmented Reality can support the connection between the physical object and the DT (Kritzler, Funk, Michahelles, \& Rohde, 2017). An authoring tool (Vuforia Studio) is connected to the IoT-Platform that transforms and contextualizes the data to create meaningful experiences.

\subsection{Cognitive Load Theory}

To optimize mental performance, the cognitive load theory has been designed to provide guidelines about the presentation of information (Sweller, Van Merrienboer, \& Paas, 1998). It was primarily developed for instructional design based on human's cognitive architecture. Without an appropriate framework of instructional techniques and knowledge about social cognitive processes, the evaluation of why instructional procedures do or do not work would be impossible (Sweller, Ayres, \& Kalyuga, 2011). From the time of its initiation, the theory generated experimental and instructional effects when new and traditional procedures were compared through randomized, controlled experiments (Sweller, 2011). In the planning of the experiment, the limited accommodation of the learner has to be taken into consideration. Therefore, the task will be divided into small steps. In addition to that, Miller (1956) demonstrated in his research that people have limited capacity for processing information and can only memorize seven elements plus/minus two in their working memory. The limited capacity of the working memory with new information is also evaluated by Sweller, Ayres, and Kalyuga (2011). Therefore, instructions and the teaching materials need to consider these limitations that information can be stored effectively in long-term memory. Because to construct them, data must be processed in the working memory (Sweller et al., 1998). Successful transformation of information into long-term memory can trivially simplify tasks that were beforehand impossible (Sweller et al., 2011).

The main interest of cognitive load theory is the ease with which information is processed in working memory. The factors which affect the working memory load are (Sweller et al., 1998): (1) intrinsic load, (2) extraneous load, and (3) germane load. Intrinsic load is described as not alterable by instructional modifications because the material being dealt with is elementary (Sweller et al., 1998). Extraneous load, on 
the other hand, is an unnecessary cognitive load and is the presented material or activities of students. Therefore, it can be adjusted by instructional interventions (Sweller et al., 1998). Germane load is a further distinction of extraneous load and reflects the effort of contribution to transfer the knowledge from working memory to long-term memory (Sweller et al., 1998). The multidimensional character and the complex interrelationships between performance, mental load, and mental effort cause difficulties of how to determine cognitive load.

Generally, the cognitive load is considered as a construct that represents the execution of a specific task afflicting on the cognitive system. This construct can be drafted in a task-based dimension (mental load) and a learner-based dimension (mental effort) while affecting the performance of the learner. Mental load refers to the load that is needed for demands of the task, and mental effort refers to the amount of cognitive capacity to accommodate the task demands (Sweller et al., 1998).

Wierwille and Eggemeier (1993) are recommending three significant categories for measuring mental-effort techniques. These classifications are including subjective, physiological, and task-performance-based indicators. The evaluation of Paas and van Merrienboer (1993) of individual and physiological measurement techniques demonstrates that physiological measures based on heart-rate variability are unreliable, invalid, and only sensitive to significant variations of cognitive load. In contrast, subjective rating-scale measurement is valid and reliable and, therefore, the most promising technique for research in the context of cognitive load theory (Sweller et al., 1998).

\section{OBJECTIVES AND METHODOLOGY}

The focus of this paper lies on reducing the cognitive load by using the Fischertechnik Simulation Unit to train students on the concept of DT and Industry 4.0. In an integrated learning scenario, the cognitive load of students working with and without a Simulation Unit will be determined and compared. This study should answer the following research question: How will the cognitive load of students be influenced by the help of simulation to understand the concept and the application of DT technology?

To answer the question, the authors plan to use the methodology of an experiment with $\mathrm{A} / \mathrm{B}$ testing. Therefore, two randomized groups will have the same problem situation, but only one will work with the Firschertechnik Simulation Unit. The measurement of the cognitive load will be performed by using the NASA task load index (NASA-TLX) (Hart, 1986; 2006). With this comparison, it can be determined which group has the lower cognitive stress level by performing the tasks necessary to understand DT.

The target group of the experiment will be students from a bachelor's degree course with no limitation in gender or age. As the authors are teaching the class on their own, it is easy to get the students for the empirical evaluation. The aim is to have 40 participants for each group in the experiment.

The main focus of the explained cognitive load theory lies in the instructional design (Sweller et al., 2011). Therefore, the approach will also be used for planning the integrated learning scenario.

\section{CONCLUSION, LIMITATIONS AND FURTHER RESEARCH}

The DT used in our paper is limited to specific implementation with the Fischertechnik 4.0. The virtual model will use the data from the sensors of the Fischertechnik Simulation Unit and is therefore limited in this context. The learning scenario will be created based on a real-world example, but it will be a simulation with a controlled environment.

This research is a first step of combining the Fischertechnik 4.0 Simulation Unit with the concept of DT to reduce the cognitive load for students. This practical approach can be used to improve the overall understanding of IT Systems over several layers (Koller et al., 2020). Parts of the outcome of the research will be used for developing a new teaching concept for the department of Management Communication \& IT. It addresses the first two layers (shopping floor, manufacturing execution) of a six-layer model for integrated ERP lectures (Dilger, Ploder \& Bernsteiner, 2020). The overall case-based teaching scenario with the DT and the Fischertechnik 4.0 can also be applied to the industry - as a showcase for digitalization. The haptic experience to see what is possible is an essential aspect for companies to indicate advantages, limitations, and potential applications. 


\section{ACKNOWLEDGEMENT}

This research is made possible by the support of the Tyrolean Science Funding.

\section{REFERENCES}

Boschert, S., \& Rosen, R. (2016). Digital twin - the simulation aspect. In Mechatronic futures (pp. 59-74). Springer.

Dilger, T., Ploder, C., \& Bernsteiner, R. (2020). "Extensive"-Case Teaching as Means of Testing Comprehensive Learning Outcome? - a Pre-Study. INTED2020 Proceedings, 1(March), 4864-4868. https://doi.org/10.21125/inted.2020.1333

Fuchs, C. (2018). Industry 4.0: The digital german ideology. Triplec: Communication, Capitalism \& Critique, 16(1), 280-289.

Grieves, M. (2015). Digital twin: Manufacturing excellence through virtual factory replication: A white paper by dr. micheal grieves.

Hart, S. G. (1986). NASA Task load Index (TLX). Volume 1.0; Paper and pencil package.

Hart, S. G. (2006, October). NASA-task load index (NASA-TLX); 20 years later. In Proceedings of the human factors and ergonomics society annual meeting (Vol. 50, No. 9, pp. 904-908). Sage CA: Los Angeles, CA: Sage Publications

Kritzler, M., Funk, M., Michahelles, F., \& Rohde, W. (2017, October). The virtual twin: controlling smart factories using a spatially-correct augmented reality representation. In Proceedings of the Seventh International Conference on the Internet of Things (pp. 1-2).

Koller, M., Ploder, C., Bernsteiner, R., \& Dilger, T. (2020). Algorithms in the IoT: An Evaluation Model for Predictive Maintenance of Cyber-Physical Systems. In L. Gaur, A. Solanki, V. Jain, \& D. Khazanchi (Eds.), Handbook of Research on Engineering Innovations and Technology Management in Organizations. https://doi.org/10.4018/978-17998-2772-6

Miller, G. A. (1956). The magical number seven, plus or minus two: Some limits on our capacity for processing information. Psychological Review, 63(2), 81.

Paas, F. G., \& Van Merriënboer, J. J. (1993). The efficiency of instructional conditions: An approach to combine mental effort and performance measures. Human factors, 35(4), 737-743.

Ploder, C., Bernsteiner, R., Schlögl, S., Gschliesser, C. (2019). The future use of LowCode / NoCode Platforms by Knowledge Workers - an Acceptance Study. In Knowledge Management in Organizations: 14th International KMO 2019, Proceedings, Cham: Springer. July 2019, doi: 10.1007/978-3-030-21451-7_38

Porter, M. E., \& Heppelmann, J. E. (2015). How smart, connected products are transforming companies. Harvard business review, 93(10), 96-114.

Rosen, R., von Wichert, G., Lo, G., \& Bettenhausen, K. (2015). About the importance of autonomy and digital twins for the future of manufacturing. ifac papers online 48 (3): 567-572. In the 15th ifac symposium on information control problems in manufacturing.

Schroeder, G., Steinmetz, C., Pereira, C. E., Muller, I., Garcia, N., Espindola, D., \& Rodrigues, R. (2016, July). Visualising the digital twin using web services and augmented reality. In 2016 IEEE 14th International Conference on Industrial Informatics (INDIN) (pp. 522-527). IEEE.

Sweller, J. (2011). Cognitive load theory. In Psychology of learning and motivation (Vol. 55, pp. 37-76). Elsevier.

Sweller, J., Ayres, P., \& Kalyuga, S. (2011). Explorations in the learning sciences, instructional systems and performance technologies, cognitive load theory. London, UK: Springer Science+ Business Media.

Sweller, J., Van Merrienboer, J. J., \& Paas, F. G. (1998). Cognitive architecture and instructional design. Educational psychology review, 10(3), 251-296.

Tao, F., Cheng, J., Qi, Q., Zhang, M., Zhang, H., \& Sui, F. (2018). Digital twin-driven product design, manufacturing, and service with big data. The International Journal of Advanced Manufacturing Technology, 94(9-12), 3563-3576.

Tao, F., Zhang, M., Cheng, J., \& Qi, Q. (2017). Digital twin workshop: a new paradigm for a future workshop. Computer Integrated Manufacturing Systems, 23(1), 1-9.

Wierwille, W. W., \& Eggemeier, F. T. (1993). Recommendations for mental workload measurement in a test and evaluation environment. Human factors, 35(2), 263-281. 